\title{
Bypass Surgery vs. Drug-Eluting Stent for Trans-Atlantic Inter-Society Consensus-II (TASCII) C or D Femoropopliteal Lesions
}

\author{
Yoshimitsu Soga, MD, PhD; Mitsuyoshi Takahara, MD, PhD; Osamu Iida, MD; \\ Shinsuke Mii, MD, PhD; Jin Okazaki, MD, PhD; Masatsugu Nakano, MD, PhD; \\ Yasutaka Yamauchi, MD, PhD; Kenji Ando, MD
}

\begin{abstract}
Background: Bypass surgery (BSX) as first-line therapy for Trans-Atlantic Inter-Society Consensus-II (TASCII) C/D femoropopliteal (FP) lesions is recommended. Recent reports have shown that a drug-eluting stent (DES) provides good durability up to the mid-term. We investigated clinical outcomes after BSX vs. DES for TASCII C/D FP lesions.

Methods and Results: As treatment of de novo TASCII C/D FP lesions, 274 patients who underwent DES implantation and 201 patients who had BSX were identified and analyzed. Each group had at least 1 year of follow-up data. The primary endpoint was binary restenosis. Secondary endpoints were major amputation, reintervention, reocclusion and major adverse limb event (MALE; including major amputation or any reintervention and restenosis). Before matching, the binary restenosis rate was significantly higher in the DES group than in the BSX group (42\% vs. $18 \%$, $\mathrm{P}<0.001)$. After propensity matching, the 1 -year restenosis rate was still higher in the DES group $(44 \%$ vs. $18 \%$, $\mathrm{P}<0.001)$. The DES group also had a significantly higher incidence of reintervention and MALE. Major amputation and reocclusion showed no significant difference. The subsequent stratification analysis reconfirmed no significant interaction effect of any background characteristics on the association of DES implantation vs. BSX with the 1-year restenosis risk.
\end{abstract}

Conclusions: BSX is still a feasible and recommended treatment for TASCII C/D FP lesions in Japanese patients, based on good durability up to 1 year. (Circ $J$ 2015; 79: 2688-2695)

Key Words: Bypass surgery; Drug-eluting stent; Femoropopliteal disease; Restenosis

$\mathbf{T}$ he Trans-Atlantic Inter-Society Consensus-II (TASCII) recommends bypass surgery (BSX) as the first-line therapy for TASCII C/D femoropopliteal (FP) lesions, instead of endovascular therapy (EVT). ${ }^{1}$ Several studies have shown that a novel bare nitinol stent decreases the restenosis rate in the short and medium term compared with conventional balloon angioplasty. ${ }^{2-5}$ However, those studies focused on TASCII A/B lesions, rather than the more complex TASCII $\mathrm{C} / \mathrm{D}$ lesions. Patency is also known to decrease for longer lesions and the efficacy of bare nitinol stent placement is not comparable to that of BSX for long lesions.

Recent reports have shown that a drug-eluting stent (DES) gives excellent short- and medium-term outcomes, ${ }^{6-8}$ and that a DES may also improve the outcomes for TASCII C/D lesions. ${ }^{9}$
However, there has not been a comparison of the outcomes of BSX and DES placement for TASCII C/D lesions. Here, we report the 1-year patency after DES placement vs. BSX using the propensity score-matching method.

\section{Study Population}

The DES group was a subgroup of the prospective multicenter ZEPHYR (ZilvEr PTX for tHe Femoral ArterY and Proximal Popliteal ArteRy) study, ${ }^{10}$ which was registered in the University Hospital Medical Information Network Clinical Trial Registry (UMIN-CTR) approved by the International Committee of Medical Journal Editors (no. UMIN000008433). The

Received June 3, 2015; revised manuscript received August 25, 2015; accepted September 7, 2015; released online October 14, 2015 Time for primary review: 26 days

Department of Cardiology (Y.S., K.A.), Department of Vascular Surgery (J.O.), Kokura Memorial Hospital, Kitakyushu; Department of Metabolic Medicine (M.T.), Department of Diabetes Care Medicine (M.T.), Osaka University Graduate School of Medicine, Suita; Cardiovascular Center, Kansai Rosai Hospital, Amagasaki (O.I.); Department of Vascular Surgery, Saiseikai Yahata General Hospital, Kitakyushu (S.M.); Division of Cardiology, Tokyo General Hospital, Tokyo (M.N.); and Cardiovascular Center, Takatsu General Hospital, Kawasaki (Y.Y.), Japan

Mailing address: Yoshimitsu Soga, MD, PhD, Department of Cardiology, Kokura Memorial Hospital, 3-2-1 Asano, Kokurakita-ku, Kitakyushu 802-0001, Japan. E-mail: sogacchy@yahoo.co.jp

ISSN-1346-9843 doi:10.1253/circj.CJ-15-0597

All rights are reserved to the Japanese Circulation Society. For permissions, please e-mail: cj@j-circ.or.jp 
ZEPHYR study included 797 limbs of 690 patients with symptomatic primary FP lesions that were treated with a Zilver ${ }^{\circledR}$ PTX $^{\circledR}$ (Cook Medical, Bloomington, IN, USA) from July 2012 to April 2013. Of these cases, 335 limbs of 297 patients had TASCII C/D lesions. The current analysis of 1-year patency was performed in 274 limbs of 242 patients, after exclusion of 27 limbs of 25 patients who died within 1 year and 34 limbs of 31 patients who dropped out within 1 year.

The bypass group in the current study comprised the bypass group of the ReCANALISE (Retrospective Comparative ANALysis of the revascularization method for Infra-inguinal arterial disease, Surgical reconstruction and Endovascular treatment) study, and was used as a historical control. The ReCANALISE study ${ }^{11,12}$ was a multicenter retrospective study including 228 limbs of 193 patients with symptomatic infrainguinal artery disease undergoing FP bypass for TASCII-II C/D lesions for the first time from January 2004 to December 2009. The current analysis of 1-year patency was performed in 201 limbs of 170 patients, after exclusion of 19 limbs of 16 patients who died within 1 year and 8 limbs of 7 patients who dropped out within 1 year.

The protocol of the current retrospective analysis was developed in accordance with the Declaration of Helsinki, and was approved by the Ethics Committee of Kansai Rosai Hospital, Amagasaki, Japan. Written informed consent was given by every patient.

\section{Procedures and Follow-up}

Endovascular and Surgical Procedures The indication for revascularization included symptomatic disease with $>50 \%$ diameter stenosis determined by angiography and mean pressure gradient $>10 \mathrm{mmHg}$ determined with a $4 \mathrm{Fr}$ diagnostic catheter. For EVT, a 6 Fr sheath was inserted into the femoral artery, mostly using a contralateral approach. After infusion of 5,000 units of heparin, a 0.035- or 0.014-inch guidewire was used to cross the lesion. Stent size was approximately $1 \mathrm{~mm}$ larger than the size of the vessel diameter assessed by angiography or intravascular ultrasound.

Dual antiplatelet therapy (DAPT) with $100-\mathrm{mg}$ /day aspirin and either $200 \mathrm{mg} /$ day ticlopidine or $75-\mathrm{mg} /$ day clopidogrel followed the manufacturer's recommendation in the package insert, starting at least 1 week before and continuing for at least 2 months after stent implantation. Other antiplatelet and anticoagulant drugs were used at each physician's discretion based on the patient's condition. Surgical revascularization was performed using standard bypass techniques. Autogenous vein or artificial bypass graft was used at each operator's discretion. Postprocedural medication was selected according to local clinical practice. Each patient was assessed for ischemic symptoms, and the ankle-brachial index was measured. Duplex ultrasound and/or CT angiography was performed to evaluate the presence of restenosis at least 1, 6 and 12 months after EVT or BSX.

\section{Outcome Measures}

The primary endpoint was the 12-month restenosis rate assessed by duplex ultrasonography, CT angiography or aortography, with a tolerance of \pm 2 months. Requirement for any reintervention or major amputation within 1 year was automatically included as a restenosis. The secondary endpoints were major amputation, reintervention, reocclusion, and major adverse limb event (MALE).

\section{Definitions}

Restenosis was defined as recurrence of $\geq 50 \%$ diameter steno- sis determined by CT angiography or aortography, or a peak systolic velocity ratio $>2.4$ on duplex ultrasonography. ${ }^{13}$ An undetectable signal in stented segments on duplex ultrasound was graded as complete occlusion. Reocclusion was defined as a target vessel that subsequently became totally occluded. Major amputation was defined as surgical excision of the limb above the ankle. Any amputation at or distal to the Lisfranc level was not considered a limb salvage failure. Amputation data were obtained through outpatient clinic follow-up. MALE was defined as major amputation or any reintervention. We defined any reintervention as any surgical or endovascular reintervention. Coronary artery disease (CAD) was defined as stable angina with evidence of coronary stenosis documented by coronary catheterization or functional or perfusion studies, history of percutaneous coronary intervention or coronary artery bypass graft surgery, or previous myocardial infarction. Cerebrovascular disease was defined as a hospital or neurologist's report of diagnosis of transient ischemic attack or ischemic stroke. Heart failure (HF) was judged to be present based on a previous diagnosis of HF, history of hospitalization for $\mathrm{HF}$, or current treatment for HF. Diabetes was defined as $\mathrm{HbA} 1 \mathrm{c}>6.5 \%$, random plasma glucose $>200 \mathrm{mg} / \mathrm{dl}$, or current treatment with oral hypoglycemic agents or insulin injection.

\section{Statistical Analysis}

Data are presented as the mean $\pm \mathrm{SD}$ for continuous variables and the number (percentage) for discrete variables, if not otherwise mentioned. A P-value $<0.05$ was considered statistically significant. We first compared the crude incidence rate of the study outcome between DES implantation and BSX. Then, 100,000-time bootstrap resampling was performed to estimate the $95 \%$ confidence intervals (CI) of the 1-year incidence of the clinical outcomes and to test the intergroup differences in incidence. To examine whether any background characteristics were influencing the prognostic effect of DES implantation vs. BSX, we performed a stratification analysis with the following background characteristics: sex, age, smoking, hypertension, dyslipidemia, diabetes mellitus, regular dialysis, cerebrovascular disease, $\mathrm{CAD}$, chronic $\mathrm{HF}$, critical limb ischemia, and TASCII-II classification. Odds ratios of DES implantation vs. BSX for 1-year restenosis and their $95 \%$ $\mathrm{CI}$ were estimated by logistic regression analysis. The $\mathrm{P}$ values for interaction between these variables and the treatment strategy were also calculated by logistic regression analysis.

To minimize intergroup differences in baseline characteristics, the 1-year clinical outcomes between DES implantation and BSX were subsequently compared after propensity scorematching. The propensity score was developed using a logistic regression model in which the following variables were entered: sex, age, smoking status, hypertension, dyslipidemia, diabetes mellitus, regular dialysis, cerebrovascular disease, $\mathrm{CAD}$, chronic HF, critical limb ischemia, and TASCII-II classification. The 1-to-1 pair matching was thereafter performed with the nearest neighbor method without replacement. According to Austin's recommendation, ${ }^{14}$ we matched on the logit of the propensity score within a caliper of $0.2 \mathrm{SD}$ of the logit of the propensity score. Intergroup differences after matching were tested by paired analysis. We performed the stratification analysis again using the matched population to reconfirm whether any baseline characteristics would influence any background characteristics would influence the prognostic effect of DES implantation vs. BSX. The odds ratios and $95 \% \mathrm{CI}$, as well as the P values for interaction were obtained from the conditional logistic regression analysis. Statistical analyses were performed using $\mathrm{R}$ version 3.1.0 (R Development Core Team, Vienna, Austria). 


\begin{tabular}{lccr|}
\hline Table 1. Baseline Characteristics of the Study Population Before Matching & & \\
& DES implantation & Bypass surgery & P value \\
$\mathrm{n}$ & 274 & 201 & \\
Age (years) & $73 \pm 8$ & $70 \pm 9$ & $<0.001$ \\
Male sex & $204(74)$ & $151(75)$ & 0.915 \\
Smoking status & & & 0.011 \\
$\quad$ Never & $87(32)$ & $53(26)$ & \\
Past & $127(46)$ & $79(39)$ & \\
Current & $60(22)$ & $69(34)$ & 0.785 \\
Hypertension & $239(87)$ & $173(86)$ & $<0.001$ \\
Dyslipidemia & $217(79)$ & $121(60)$ & 0.254 \\
Diabetes mellitus & $174(64)$ & $117(58)$ & 0.001 \\
Regular dialysis & $75(27)$ & $29(14)$ & $<0.001$ \\
Cerebrovascular disease & $54(20)$ & $73(36)$ & 0.163 \\
Coronary artery disease & $152(55)$ & $98(49)$ & 0.094 \\
Chronic heart failure & $49(18)$ & $24(12)$ & 0.008 \\
Fontaine classification & & & \\
$\quad I^{\circ}$ (intermittent claudication) & $194(71)$ & $122(61)$ & $<0.001$ \\
III' (rest pain) & $30(11)$ & $18(9)$ & \\
IV (tissue loss) & $50(18)$ & $61(30)$ & $170(85)$ \\
TASCII class D & $166(61)$ & & \\
\hline
\end{tabular}

Data are mean and SD, or $\mathrm{n}(\%)$. Intergroup difference was tested by unpaired t-test for continuous variables, by Fisher's exact test for dichotomous variables, and by Mann-Whitney $U$ test for the other discrete variables. DES, drug-eluting stent; TASCII, Trans-Atlantic Inter-Society Consensus-II.

\section{Crude one-year incidence after revascularization in the overall study population}

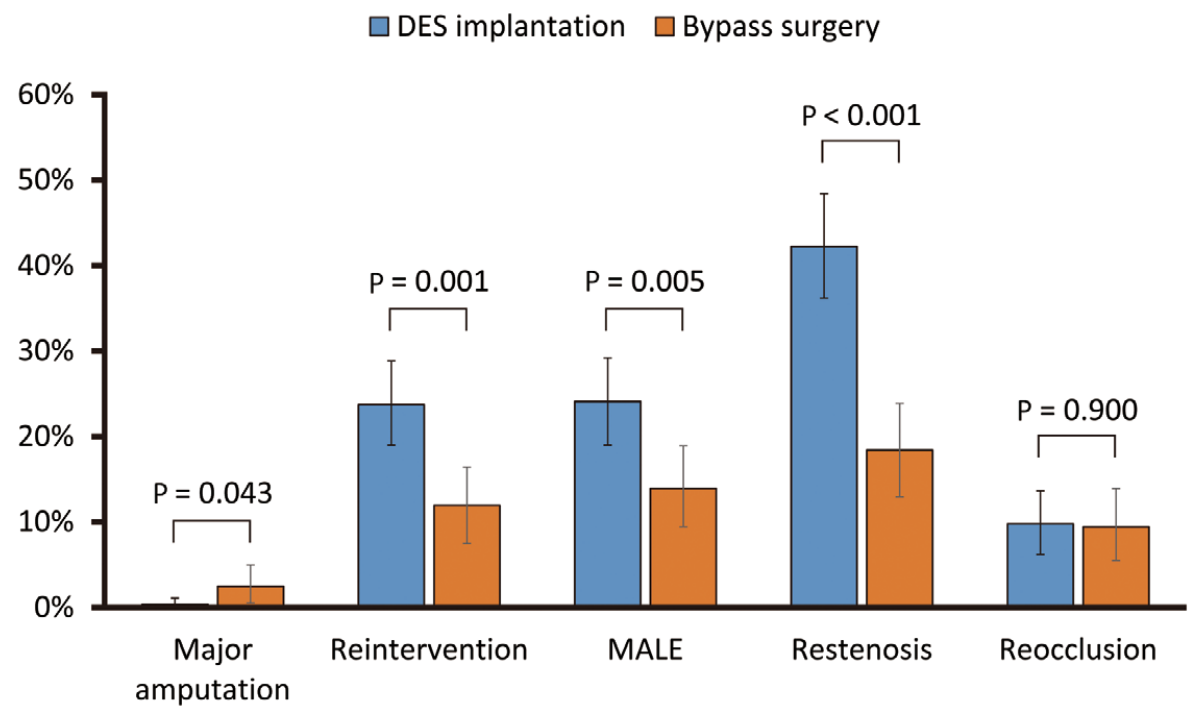

Figure 1. Crude 1-year clinical outcomes after femoropopliteal revascularization for Trans-Atlantic Inter-Society Consensus-II (TASCII) class C/D lesions in the overall study population. Error bars represent 95\% confidence intervals. DES, drug-eluting stent; MALE, major adverse limb event.

\section{Results}

A total of 274 cases of DES implantation and 201 cases of BSX were included in the current study. Table 1 shows the baseline characteristics of the study population. Compared with the BSX group, the DES-implanted group were older and a higher prevalence of smoking, dyslipidemia, and regular dialysis, whereas the BSX group was more likely to have cerebrovascular disease, critical limb ischemia, and TASCII class D. In the BSX group, 65 lesions (32\%) were treated with 


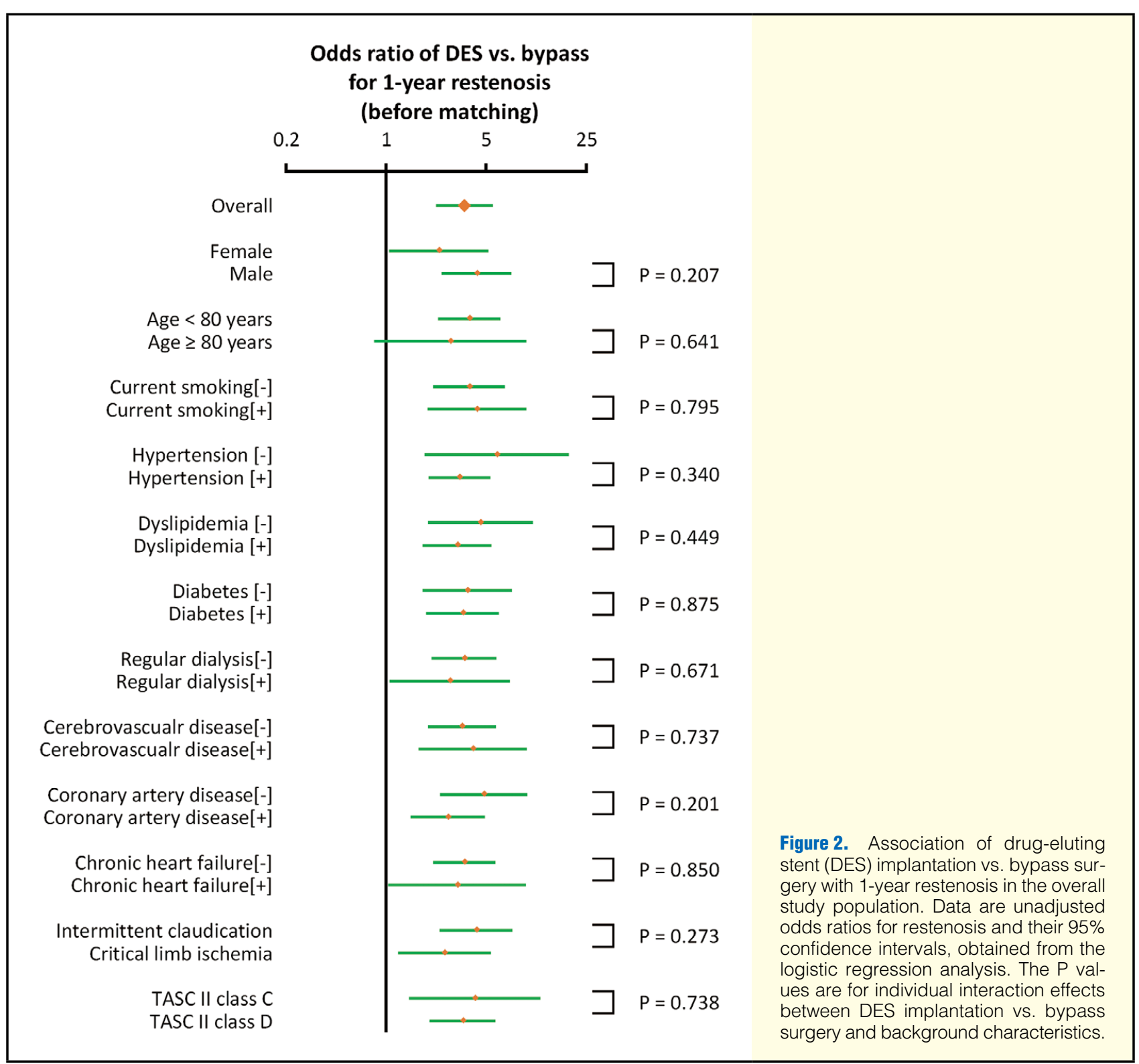

vein grafts. Mean number of DES used was $2.4 \pm 0.7$ stents per lesion ( 1 stent in 29 cases, 2 stents in 106 cases, 3 stents in 128 cases and 4 stents in 11 cases). In this study, 201 limbs underwent BSX and of them, 174 patients underwent AKFP bypass (anastomosed above the knee joint) and 27 underwent BKFP bypass (anastomosed below the knee joint). Distal bypass was not included.

Figure 1 shows the comparison of the crude 1-year incidence of the study outcomes between the DES and BSX groups. The DES group had a significantly lower incidence of major amputation, but a significantly higher incidence of reintervention, MALE, and restenosis. The 1-year restenosis rate was $42 \%$ (95\% CI: $3-48 \%$ ) in the DES group and 18\% (95\% CI: $13-24 \%)$ in the BSX group $(\mathrm{P}<0.001)$. As shown in Figure 2, the subsequent stratification analysis demonstrated that no background characteristics had a significant influence on the association of DES implantation vs. BSX with restenosis risk (all $\mathrm{P}$ for interaction $>0.05$ ).

We therefore performed propensity score-matching without any stratification of the study population. The matching extracted 147 pairs (Figure 3 ). There was no significant intergroup difference in baseline characteristics after matching in both subgroups (Table 2).

The 1-year incidence rate of the study outcomes in the matched population is shown in Figure 4. The DES group had a significantly higher incidence of reintervention, MALE, and restenosis. The 1-year restenosis rate was $44 \%$ (95\% CI: $36-53 \%$ ) in the DES group and $18 \%$ (95\% CI: 12-24\%) in the BSX group $(\mathrm{P}<0.001)$. Major amputation showed no significant intergroup difference after the matching. The subsequent stratification analysis reconfirmed no significant interaction effect of any background characteristics on the association of DES implantation vs. BSX with 1-year restenosis risk (Figure 5).

\section{Discussion}

This study compared 1-year outcomes between patients with 


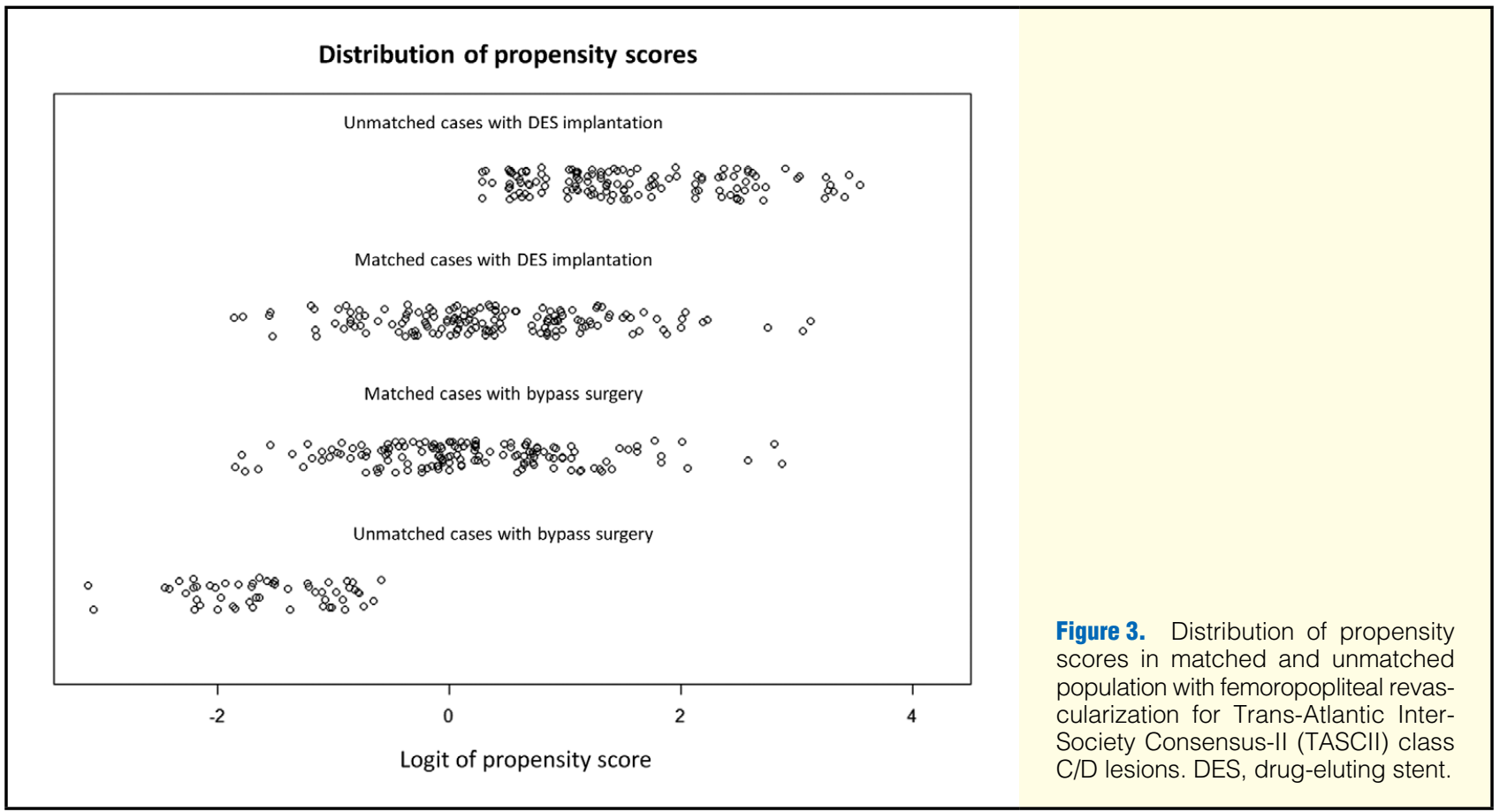

\begin{tabular}{|lccc|}
\hline Table 2. Baseline Characteristics of the Matched Study Population & & \\
$\mathrm{n}$ & DES implantation & Bypass surgery & P value \\
Age (years) & 147 & 147 & 0.631 \\
Male sex & $72 \pm 9$ & $71 \pm 8$ & 0.899 \\
Smoking status & $109(74)$ & $107(73)$ & 0.891 \\
$\quad$ Never & & & \\
Past & $43(29)$ & $42(29)$ & \\
Current & $59(40)$ & $63(43)$ & 0.868 \\
Hypertension & $45(31)$ & $42(29)$ & 0.161 \\
Dyslipidemia & $125(85)$ & $127(86)$ & 0.724 \\
Diabetes mellitus & $112(76)$ & $101(69)$ & 1.000 \\
Regular dialysis & $93(63)$ & $89(61)$ & 0.795 \\
Cerebrovascular disease & $25(17)$ & $25(17)$ & 0.822 \\
Coronary artery disease & $37(25)$ & $40(27)$ & 0.607 \\
Chronic heart failure & $79(54)$ & $76(52)$ & 0.997 \\
Fontaine classification & $21(14)$ & $17(12)$ & \\
II (intermittent claudication) & & & \\
III' (rest pain) & $102(69)$ & $100(68)$ & $18(12)$ \\
IV (tissue loss) & $15(10)$ & $29(20)$ & 0.200 \\
TASCll class D & $30(20)$ & $118(80)$ & \\
\hline
\end{tabular}

Data are means and SD, or $\mathrm{n}(\%)$. Intergroup difference was tested by paired t-test for continuous variables, by the McNemar test for dichotomous variables, and by the Wilcoxon signed rank test for the other discrete variables. Abbrevistions as in Table 1.

femoropopliteal lesions treated with DES placement or BSX. The use of a DES for prevention of restenosis in short lesions has been reported in Japanese patients ${ }^{8}$ and resulted in improved outcomes in patients with TASCII C/D lesions, compared with conventional therapies. Therefore, we expected that use of a DES might be a replacement for BSX. However, our results showed that the restenosis and revascularization rates were significantly higher after DES placement compared with BSX.

Unlike a previous report, ${ }^{9}$ our results indicated that prevention of restenosis using current DES is relatively ineffective for TASCII C/D lesions. Why were the results for DES not as good as expected? There are several possible reasons. Firstly, the DES group seemed to include more severe lesions and higher-risk patient backgrounds such as female sex, dialysis patients, diabetic patients, ischemic severity, poor runoff ${ }^{15}$ and 


\section{One-year incidence after revascularization in the matched population}

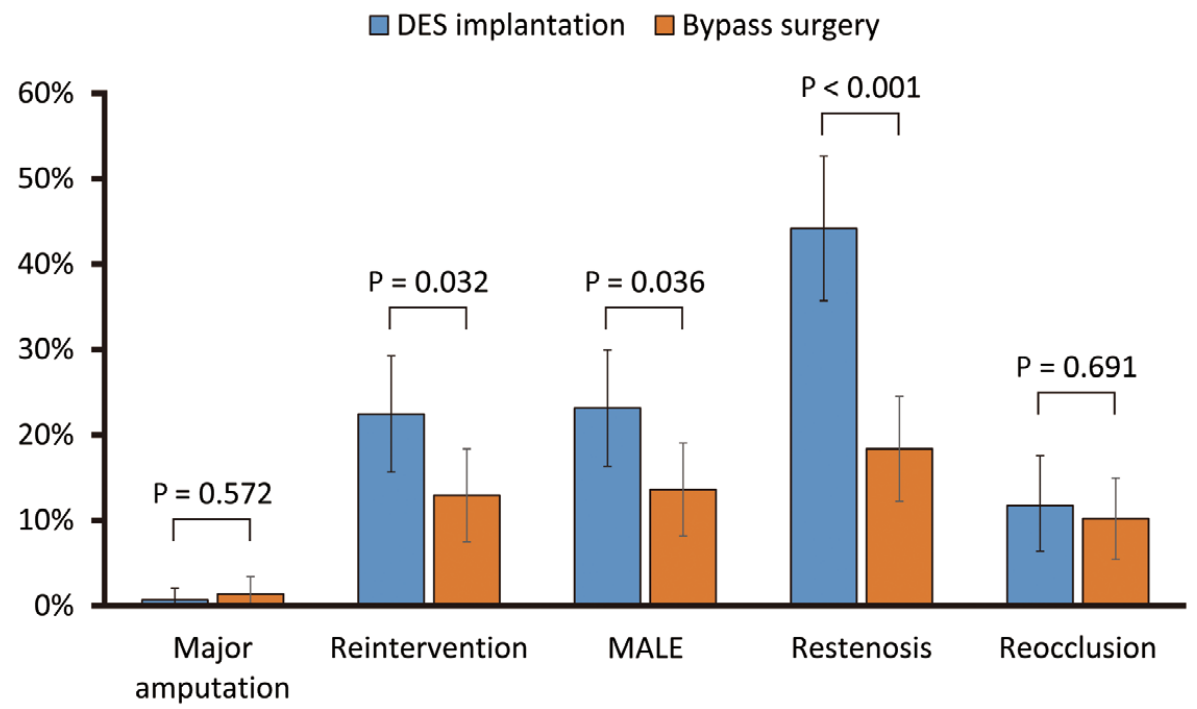

Figure 4. One-year clinical outcomes after femoropopliteal revascularization for Trans-Atlantic Inter-Society Consensus-II (TASCII) class C/D lesions in the matched population. Error bars represent 95\% confidence intervals. DES, drug-eluting stent; MALE, major adverse limb event.

small vessels. In particular, heavily calcified lesions in dialysis patients might influence the effect of the DES, and contribute to the chronic result. Secondly, although the previous paper ${ }^{9}$ reported good durability of the Zilver PTX for TASCII C/D and restenotic lesions (12-month primary patency; $85.5 \%$ ), the follow-up duration was shorter (mean 5.3 months; range 1-24 months) and the sample size of TASCII C/D patients was smaller $(n=33)$. These differences may affect the results. In addition, the maximal stent length is limited, which requires several stents to cover the whole lesion. Many overlaps or chronic outward force might also influence the increase in restenosis of longer lesions in the chronic phase. Because of compliance with the DAPT protocol for at least 2 months, the drugs were unlikely to influence restenosis and reocclusion. A study of bypass grafts showed less satisfactory results with synthetic polytetrafluoroethylene grafts compared with venous grafts, ${ }^{1}$ but in the current study population there were no statistically significant differences in endpoints at 1 year (restenosis, reocclusion, MALE, major amputation) between synthetic polytetrafluoroethylene-coated grafts and venous grafts (data not shown).

The restenosis rates in the BSX group before and after matching were $18 \%$ [95\% CI: $13-24 \%$ ] and $18 \%$ [95\% CI: 12-24\%], respectively, and the $99 \%$ CI before and after matching were $15-26 \%$ and $14-27 \%$, respectively. These results suggested that a 1-year restenosis rate of at least $20-25 \%$ with DES implantation is required to show that this method has similar efficacy to BSX for TASCII-II C/D lesions. A 1-year restenosis rate of less than $30 \%$ might be acceptable, given the minimal invasion and shorter hospital stay with EVT.

We previously reported that the 1-year restenosis rate after EVT (balloon angioplasty with an optional bare nitinol stent) for TASCII-II C/D lesions in claudicant patients was estimated as $32 \%$ by the Kaplan-Meier method. ${ }^{11}$ The 1 -year restenosis rate after DES implantation in the current study might be apparently higher compared with that report. However, the current analysis did not use Kaplan-Meier estimation in order not to overlook restenotic events up to 14 months, within the prespecified tolerance of 1-year patency assessment. This stricter evaluation would cause an apparently higher rate compared with the previous Kaplan-Meier estimation. In addition, we also note that approximately $30 \%$ of the patients had severe ischemic limbs, which may explain the higher restenosis rate compared with the previous study.

The findings in this study were obtained in clinical settings. Thus, to decrease the restenosis rate, evaluation of the effects and limitations of DES in clinical settings is required, including studies on improved mimetic stent design, such as use of the Supera stent ${ }^{16}$ (IDEV Technologies, Inc, Webster, TX, USA) as a platform. Recent reports have shown excellent outcomes with drug-coated balloons for short lesions, ${ }^{17,18}$ and this approach may also be effective for long and complex lesions. A comparison study is also required using a covered stent, ${ }^{19}$ with which the restenosis rate may not be significantly affected by lesion length. It is also currently difficult to achieve good outcomes with a single device, and further studies are needed to examine the use of combinations of devices and optimization of these devices for treatment of longer, complex FP lesions.

Finally, the EVT procedure was repeatable and less invasive despite higher restenosis compared with BSX. BSX was more invasive, but longer-term durability after successful surgery could be expected. Our results did not support using BSX for all patients with complex FP lesions. Treatment strategy should be comprehensively based on lesion characteristics in addition to age, general status, frailty, comorbidities, runoff vessel, vessel diameter, the presence of a durable vein and 


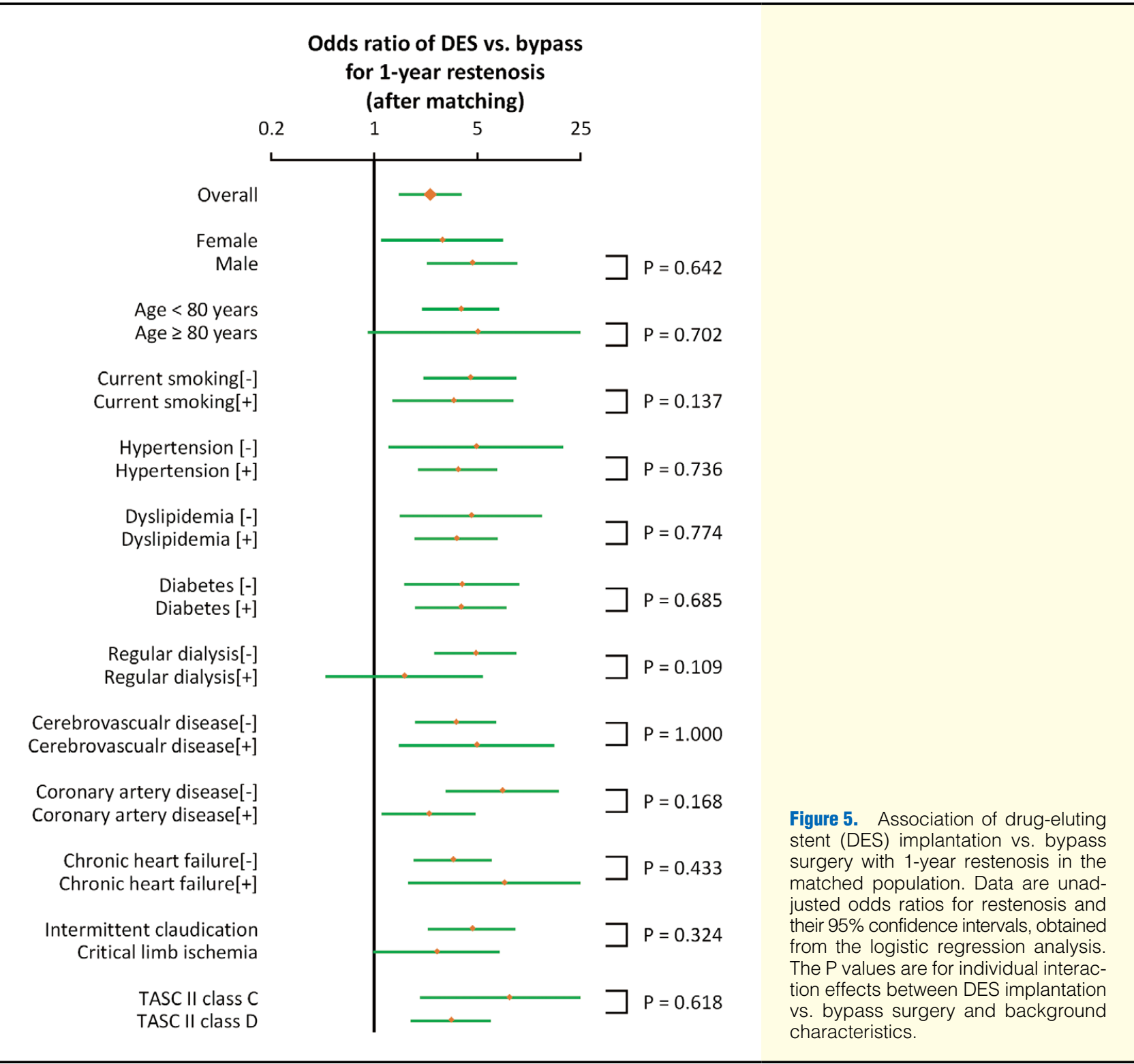

many other factors. Although restenosis is one of the many clinical endpoints, it is not the definitive factor for treatment selection.

\section{Study Limitations}

First, we used historical data for the BSX group, despite this being a multicenter study and a propensity score-matched analysis. Unlike prospective data, the restenosis rate in the BSX group might be underestimated because of retrospective data collection. To minimize any influence on outcome, we selected surgical facilities that strictly performed routine follow-up for at least 1 year and enrolled consecutive cases. Therefore, a prospective randomized clinical trial is required to verify our findings. Second, although the adjusted factors were selected and seemed clinically reasonable, these variables were arbitrary. Additionally, the follow-up period was short, making it hard to evaluate durability and feasibility. Longer-term follow-up is needed to confirm these results. Third, the study was performed in Japanese patients, and racial dif- ferences in outcomes require evaluation. Also, the study period was different between the ReCANALISE and ZEPHYR trials. EVT for SFA lesions was still not performed frequently compared with recent years. Therefore, in TASC C lesions such as restenotic lesions, it may not be similar in the current era. Accumulation of cases is needed to verify these possible biases. Finally, the conclusions might not be applicable to patients with anatomic complexities such as calcification or vessel runoff, which were not evaluated in this study. In addition, low use of autologous vein grafts may affect clinical outcome. Possible factors, however, were adjusted in the propensity-matched analysis.

\section{Conclusions}

Our result suggested that BSX is still feasible as a treatment of TASCII C/D FP disease in Japanese patients, based on good durability up to 1 year, when compared with the use of a firstgeneration DES (Zilver PTX). However, in the clinical setting, 
the indication and strategy for revascularization should be carefully decided, taking into consideration lesion characteristics, the patient's status, the presence of a durable vein and other clinical factors.

\section{Financial Support}

None.

\section{Conflict of Interest}

None declared.

\section{References}

1. Norgren L, Hiatt WR, Dormandy JA, Nehler MR, Harris KA, Fowkes FG; TASCII II Working Group. Inter-Society Consensus for the Management of Peripheral Arterial Disease (TASCII II). $J$ Vasc Surg 2007; 45(Suppl S): S5-S67.

2. Dick P, Wallner H, Sabeti S, Loewe C, Mlekusch W, Lammer J, et al. Balloon angioplasty versus stenting with nitinol stents in intermediate length superficial femoral artery lesions. Catheter Cardiovasc Interv 2009; 74: 1090-1095.

3. Schillinger M, Sabeti S, Loewe C, Dick P, Amighi J, Mlekusch W, et al. Balloon angioplasty versus implantation of nitinol stents in the superficial femoral artery. N Engl J Med 2006; 354: 1879-1888.

4. Zeller T, Tiefenbacher C, Steinkamp HJ, Langhoff R, Wittenberg G, Schlüter M, et al. Nitinol stent implantation in TASCII A and B superficial femoral artery lesions: The Femoral Artery Conformexx Trial (FACT). J Endovasc Ther 2008; 15: 390-398.

5. Laird JR, Katzen BT, Scheinert D, Lammer J, Carpenter J, Buchbinder M, et al. Nitinol stent implantation versus balloon angioplasty for lesions in the superficial femoral artery and proximal popliteal artery: Twelve-month results from the RESILIENT randomized trial. Circ Cardiovasc Interv 2010; 3: 267-276.

6. Dake MD, Ansel GM, Jaff MR, Ohki T, Saxon RR, Smouse HB, et al. Paclitaxel-eluting stents show superiority to balloon angioplasty and bare metal stents in femoropopliteal disease: Twelve-month Zilver PTX randomized study results. Circ Cardiovasc Interv 2011; 4: $495-504$.

7. Dake MD, Ansel GM, Jaff MR, Ohki T, Saxon RR, Smouse HB, et al. Sustained safety and effectiveness of paclitaxel-eluting stents for femoropopliteal lesions: 2-year follow-up from the Zilver PTX randomized and single-arm clinical studies. J Am Coll Cardiol 2013; 61: $2417-2427$

8. Ohki T, Yokoi H, Kichikawa K, Kimura T, Snyder SA, Ragheb AO, et al. Two-year analysis of the Japanese cohort from the Zilver PTX randomized controlled trial supports the validity of multinational clinical trials. J Endovasc Ther 2014; 21: 644-653.

9. Leopardi M, Houbballah R, Becquemin JP. Effectiveness of Zilver PTX eluting stent in TASCII C/D lesions and restenosis. $J$ Cardiovasc Surg 2014; 55: 229-234.

10. Iida O, Takahara M, Soga Y, Nakano M, Yamauchi Y, Kan Z, et al. 1-year results of the ZEPHYR (ZilvEr PTX for tHe Femoral ArterY and Proximal Popliteal ArteRy) Registry: Predictors of restenosis. JACC Cardiovasc Interv 2015; 8: 1105-1112.

11. Aihara H, Soga Y, Mii S, Okazaki J, Yamaoka T, Kamoi D, et al. Comparison of long-term outcome after endovascular therapy versus bypass surgery in claudication patients with Trans-Atlantic InterSociety Consensus-II C and D femoropopliteal disease. Circ J 2014; 78: $457-464$.

12. Soga Y, Mii S, Aihara H, Okazaki J, Kuma S, Yamaoka T, et al. Comparison of clinical outcome after bypass surgery vs. endovascular therapy for infrainguinal artery disease in patients with critical limb ischemia. Circ J 2013; 77: 2102-2109.

13. Ranke C, Creutzig A, Alexander K. Duplex scanning of the peripheral arteries: Correlation of the peak velocity ratio with angiographic diameter reduction. Ultrasound Med Biol 1992; 18: 433-440.

14. Austin PC. Optimal caliper widths for propensity-score matching when estimating differences in means and differences in proportions in observational studies. Pharm Stat 2011; 10: 150-161.

15. Soga Y, Iida O, Hirano K, Suzuki K, Tosaka A, Yokoi H, et al. Utility of new classification based on clinical and lesional factors after self-expandable nitinol stenting in the superficial femoral artery. $J$ Vasc Surg 2011; 54: 1058-1066.

16. Werner M, Paetzold A, Banning-Eichenseer U, Scheinert S, Piorkowski M, Ulrich M, et al. Treatment of complex atherosclerotic femoropopliteal artery disease with a self-expanding interwoven nitinol stent: Midterm results from the Leipzig SUPERA 500 registry. EuroIntervention 2014; 10: $861-868$.

17. Tepe G, Laird J, Schneider P, Brodmann M, Krishnan P, Micari A, et al. Drug-coated balloon versus standard percutaneous transluminal angioplasty for the treatment of superficial femoral and popliteal peripheral artery disease: 12 -month results from the IN.PACT SFA randomized trial. Circulation 2015; 131: 495-502.

18. Liistro F, Grotti S, Porto I, Angioli P, Ricci L, Ducci K, et al. Drugeluting balloon in peripheral intervention for the superficial femoral artery: The DEBATE-SFA randomized trial (drug eluting balloon in peripheral intervention for the superficial femoral artery). JACC Cardiovasc Interv 2013; 6: 1295-1302.

19. Lammer J, Zeller T, Hausegger KA, Schaefer PJ, Gschwendtner M, Mueller-Huelsbeck S, et al. Sustained benefit at 2 years for covered stents versus bare-metal stents in long SFA lesions: The VIASTAR Trial. Cardiovasc Intervent Radiol 2015; 38: 25-32. 